\title{
Three-dimensional facial development of children with unilateral cleft lip and palate during the first year of life in comparison with normative average faces
}

\author{
Sander Brons ${ }^{\text {Corresp., }}{ }^{1}$, Jene W Meulstee ${ }^{2}$, Tom GJ Loonen ${ }^{2}$, Rania M Nada ${ }^{3}$, Mette AR Kuijpers ${ }^{1}$, Ewald M \\ Bronkhorst $^{4}$, Stefaan J Bergé ${ }^{5}$, Thomas JJ Maal ${ }^{2}$, Anne Marie Kuijpers-Jagtman ${ }^{1}$ \\ 1 Department of Dentistry, Section of Orthodontics and Craniofacial Biology, Radboud University Medical Centre, Nijmegen, The Netherlands \\ 2 Department of Oral and Maxillofacial Surgery, Radboudumc 3D Lab, Radboud University Medical Centre, Nijmegen, The Netherlands \\ 3 Faculty of Dentistry, Kuwait University, Kuwait City, Kuwait \\ 5 Department of Oral and Maxillofacial Surgery, Radboud University Medical Centre, Nijmegen, The Netherlands \\ Corresponding Author: Sander Brons \\ Email address: sander.brons@radboudumc.nl
}

Background. Stereophotogrammetry can be used to study facial morphology in both healthy individuals as well as subjects with orofacial clefts because it shows good reliability, ability to capture images rapidly, archival capabilities, and high resolution, and does not require ionizing radiation. This study aimed to compare the 3D facial morphology of infants born with unilateral cleft lip and palate (UCLP) with an age-matched normative 3D average face before and after primary closure of the lip and soft palate.

Methods. Thirty infants with a non-syndromic complete unilateral cleft lip, alveolus, and palate participated in the study. 3D images were acquired at 3, 6, 9, and 12 months of age. All subjects were treated according to the primary surgical protocol consisting of surgical closure of the lip and the soft palate at 6 months of age. 3D images of UCLP patients at 3, 6 (pre-treatment), 9, and 12 months of age were superimposed on normative datasets of average facial morphology using the children's reference frame. Distance maps of the complete 3D facial surface and the nose, upper lip, chin, forehead, and cheek regions were developed.

Results. Assessments of the facial morphology of UCLP and control subjects by using color-distance maps showed large differences in the upper lip region at the location of the cleft defect and an asymmetry at the nostrils at 3 and 6 months of age. At 9 months of age, the labial symmetry was completely restored although the tip of the nose towards the unaffected side showed some remnant asymmetry. At 12 months of age, the symmetry of the nose improved, with only some remnant asymmetry noted on both sides of the nasal tip. At all ages, the mandibular and chin regions of the UCLP patients were $2.5-5 \mathrm{~mm}$ posterior to those in the average controls.

Conclusion. In patients with UCLP deviations from the normative average 3D facial morphology of agematched control subjects existed for the upper lip, nose, and even the forehead before lip and soft palate closure was performed. Compared to the controls symmetry in the upper lip was restored, and the shape of the upper lip showed less variation after primary lip and soft palate closure. At this early age, retrusion of the soft-tissue mandible and chin, however, seems to be developing already. 


\section{Three-dimensional facial development of children with}

\section{2 unilateral cleft lip and palate during the first year of life in \\ 3 comparison with normative average faces}

4 Short title: 3D facial development in unilateral cleft lip and palate

6 Sander Brons ${ }^{1}$, Jene W. Meulstee ${ }^{2}$, Tom G.J. Loonen ${ }^{2}$, Rania M. Nada ${ }^{4}$, Mette A.R. Kuijpers ${ }^{1}$,

7 Ewald M. Bronkhorst ${ }^{5}$, Stefaan J. Bergé ${ }^{5}$, Thomas J.J. Maal ${ }^{2}$, Anne Marie Kuijpers-Jagtman ${ }^{1}$

$9 \quad{ }^{1}$ Department of Dentistry, Section of Orthodontics and Craniofacial Biology, Radboud

10 University Medical Centre, Philips van Leydenlaan 25, 6525EX Nijmegen, Netherlands

${ }^{2}$ Department of Oral and Maxillofacial Surgery, Radboudumc 3D Lab, Radboud University

Medical Centre, Geert Grooteplein Zuid 10, 6525GA Nijmegen, Netherlands

${ }^{3}$ Faculty of Dentistry, Kuwait University, Street 102, Jabriya Kuwait City, Kuwait

${ }^{4}$ Department of Dentistry, Section of Preventive and Curative Dentistry, Radboud University

Medical Centre, Philips van Leydenlaan 25, 6525EX Nijmegen, Netherlands

${ }^{5}$ Department of Oral and Maxillofacial Surgery, Radboud University Medical Centre, Geert Grooteplein Zuid 10, 6525GA Nijmegen, Netherlands

Corresponding author: Sander Brons

Department of Dentistry, Section of Orthodontics and Craniofacial Biology, Radboud University

ORCID ID: 0000-0001-5263-4804 


\section{Abstract}

Background. Stereophotogrammetry can be used to study facial morphology in both healthy individuals as well as subjects with orofacial clefts because it shows good reliability, ability to capture images rapidly, archival capabilities, and high resolution, and does not require ionizing radiation. This study aimed to compare the 3D facial morphology of infants born with unilateral cleft lip and palate (UCLP) with an age-matched normative 3D average face before and after primary closure of the lip and soft palate.

Methods. Thirty infants with a non-syndromic complete unilateral cleft lip, alveolus, and palate participated in the study. 3D images were acquired at 3, 6, 9, and 12 months of age. All subjects were treated according to the primary surgical protocol consisting of surgical closure of the lip and the soft palate at 6 months of age. 3D images of UCLP patients at 3, 6 (pretreatment), 9, and 12 months of age were superimposed on normative datasets of average facial morphology using the children's reference frame. Distance maps of the complete 3D facial surface and the nose, upper lip, chin, forehead, and cheek regions were developed.

Results. Assessments of the facial morphology of UCLP and control subjects by using color-distance maps showed large differences in the upper lip region at the location of the cleft defect and an asymmetry at the nostrils at 3 and 6 months of age. At 9 months of age, the labial symmetry was completely restored although the tip of the nose towards the unaffected side showed some remnant asymmetry. At 12 months of age, the symmetry of the nose improved, with only some remnant asymmetry noted on both sides of the nasal tip. At all ages, the mandibular and chin regions of the UCLP patients were $2.5-5 \mathrm{~mm}$ posterior to those in the average controls.

Conclusion. In patients with UCLP deviations from the normative average 3D facial morphology of age-matched control subjects existed for the upper lip, nose, and even the forehead before lip and soft palate closure was performed. Compared to the controls symmetry in the upper lip was restored, and the shape of the upper lip showed less variation after primary lip and soft palate closure. At this early age, retrusion of the soft-tissue mandible and chin, however, seems to be developing already. 


\section{Introduction}

54 Orofacial clefts (OFCs) are one of the most frequently diagnosed congenital craniofacial malformations ( $\mathrm{WHO}, 2002$; Kadir et al., 2017). OFCs lead to several problems such as impairment of facial and dental development, speech and hearing, and facial esthetics. Therefore, affected individuals are liable to suffer stigmatization, social exclusion, and barriers to employment (WHO, 2002). To maximize their potential in adolescent and adult life, affected individuals need the care of an interdisciplinary team of specialists (Shaw et al., 2001; Ness et al., 2015; $A C P C A, 2016$; ).

Treatment of OFCs starts within the first months after birth. Primary treatment of a complete unilateral or bilateral cleft may include presurgical infant orthopedics (plate and/or taping of the lip and/or nasoalveolar molding) and primary surgical correction of the cleft lip and palate. Different techniques and timelines have been described for infant orthopedics and cleft lip closure. The use of numerous different protocols for primary treatment of OFCs across different treatment centers indicates the lack of clear scientific evidence favoring one method over the others (Shaw et al., 2000).

Facial morphology is an important outcome variable of cleft treatment, and various methods for assessment of facial morphology have been described in the literature, including direct physical measurements (Farkas, Hajnik \& Poswick, 1993; Reddy et al., 2010), rating of standardized clinical photographs (Chowdri, Darzi \& Ashraf, 1990; Halli et al., 2012), as well as sophisticated three-dimensional (3D) imaging techniques (Kuijpers et al., 2014). A systematic review of the literature regarding the reliability and application of 3D facial imaging methods in babies and young children, both unaffected control subjects as well as subjects with OFCs, concluded that stereophotogrammetry is the preferred method due to its millisecond fast image capture, archival capabilities, high resolution, and no exposure to ionizing radiation (Brons et al., 2012). However, only a few studies have used (3D) stereophotogrammetry for evaluation of facial growth and treatment outcome in infants with OFCs until 12 months of age. Two studies used linear and angular measurements for evaluation of facial morphology before and after primary lip closure (Alazzawi et al., 2017; Morioka et al., 2018), and one study also made a comparison with the facial morphology of a control sample (Mancini et al., 2018). Furthermore, one study used superimposition of individual 3D images for evaluation of facial morphology before and after 
83

84

85

86

87

88

89

90

91

92

101

102

103

104

105

106

107

108

109

110

nasoalveolar molding and primary cheilorhinoplasty (Wu et al., 2016). One recent study used superimposition of 3D images with generic meshes for evaluation of facial morphology before and after primary cheilorhinoplasty (Al-Rudainy et al., 2018). The use of a generic mesh is a recent advancement in technical analysis of 3D facial images. It allows better utilization of the potential of complex longitudinal 3D information than simple linear and angular measurements, removes the need for localization of landmarks and thus reduces operator error, and allows automatic identification of corresponding facial regions by superimposing 3D images with a generic mesh (Brons 2018).

Comparison of the facial morphology of individuals with craniofacial malformations and normal controls can be achieved by registration of average faces of each group (Hammond et al., 2004). Construction of average faces from 3D images of patients with OFC and controls aged 8 to 12 years has been reported earlier (Bugaighis et al., 2014). To our knowledge, there is no published report on facial growth and treatment outcome in infants with OFCs and controls matched for age during the first year of life using stereophotogrammetry and superimposition with the use of a generic mesh and average faces. Therefore, the aim of this study was to compare the 3D facial morphology of infants born with unilateral cleft lip and palate (UCLP) with the normative average $3 \mathrm{D}$ facial morphology of age-matched control subjects before and after primary closure of the lip and soft palate with the application of a generic mesh.

\section{Materials \& Methods}

\section{Ethical approval and informed consent}

The medical ethics commission of the institution in which the study was conducted approved the study protocol (Commissie Mensgebonden Onderzoek regio Arnhem-Nijmegen \#2007/163). The study was performed in accordance with the 1964 Declaration of Helsinki and its subsequent amendments. Written informed consent from the subjects' parents was obtained prior to their inclusion in the study.

Subjects

Peer) reviewing PDF | (2019:03:35537:1:1:NEW 20 May 2019) 
111 The patients in this study were children with non-syndromic complete UCLP. The subjects in

112 this study are part of a prospective longitudinal 3D study on facial growth from the age of 3

113 months to 6 years (Brons et al., 2018). Data for patients with UCLP were collected within the

114 first month after birth at the Cleft Palate Craniofacial Unit of the Radboud University Medical

115 Centre in Nijmegen, the Netherlands, between September 2008 and December 2011. The

116 inclusion criteria were non-syndromic complete UCLP and age $\leq 3$ months at the time of study

117 entry. Only infants born at term $(38+$ wk) to parents who were both Caucasians were included.

118 Exclusion criteria were congenital malformations other than UCLP and the presence of soft-

119 tissue bands. A total of 30 patients with UCLP were enrolled in the study.

120 The control group was recruited before the age of 3 months at the Maternity Clinic of the

121 Radboud University Medical Centre and Regional Health Services (GGD Gelderland-Zuid)

122 between April 2007 and September 2010. Inclusion criteria for the controls were born at term

$123(38+w k)$ and both parents Caucasian. Exclusion criteria for the controls were occurrence of oral

124 clefts in the first-, second-, or third-degree relatives. Fifty controls were enrolled in the study. In

125 a previous study, normative average faces at 3, 6, 9 and 12 months of age were developed (Brons

126 et al., 2018).

127

128 Treatment protocol

129 Since 2008, the primary surgery protocol at the Nijmegen Cleft Palate Craniofacial Unit consists

130 of surgical closure of the lip and the soft palate at 6 months of age. The lip is closed by a

131 modified Millard technique, and closure of the soft palate is done according to a modified von

132 Langenbeck technique. The hard palate is closed at 3 years of age, and alveolar bone grafting is

133 performed at 9-11 years of age before eruption of the permanent canine.

134

1353 image acquisition

136 Acquisition of 3D images was done within a period of -21 and +21 days around the age of 3,6 ,

1379 , and 12 months. Image acquisition was performed in a designated 3D imaging room with no

138 windows and a consistent amount of ambient lighting. The 3dMDfacial System (3dMD Ltd., 
139 Atlanta, GA, USA) with a 2-pod configuration was used for frontal facial image acquisition

140 including both ears. The distance between the infants and camera's was 1 meter. The camera was

141 calibrated on a daily basis. The 3D images consisted of approximately 20.000 points and the

142 texture map was 8 megapixel. 3D images of the subjects' faces were acquired at 3, 6 (pre-

143 treatment), 9, and 12 months of age at the 3D Lab (Nijmegen, Netherlands) by trained

144 photographers. The duration of the image capture was 1.5 milliseconds. On each occasion,

145 approximately three images were obtained within 10 minutes. Image quality was visually

146 assessed immediately after acquisition by the photographer for completeness of 3D image data

147 and a neutral facial expression using 3dMDpatient V4.0 software.

\section{Selection of eligible $3 D$ images}

150

151

152

153

154

155

156

157

158

159

160

161

162

163

164

165

166

167

High-quality 3D images of the face at rest from the entire sample at 3, 6, 9, and 12 months of age were selected. Inclusion criteria were (1) a neutral facial expression with the eyes open and the lips lightly opposed without straining, (2) orientation of the face in the natural head position, (3) no data holes in the facial region medial to the ears, caudal to the hairline, and cranial to the menton, (4) correct 3D image construction, and (5) no presence of lip tape or a nasal feeding tube. The reliability of the selection procedure was very good, as shown by a kappa value of 0.90 (Brons et al., 2018).

\section{$3 D$ image processing}

The selected 3D images were exported from the 3dMDpatient 4.0 software as wavefront object (.obj) files with texture. Next, the 3D images were imported into Maxilim version 2.3.0.3

(Nobel-Biocare, Mechelen, Belgium). The children's reference frame described previously was used to align all 3D images in the correct position and orientation (Brons et al., 2013). Third, 3D images of the right-sided UCLP subjects were mirrored on the mid-sagittal plane to obtain 3D images of left-sided UCLP only. Next, remeshing of the 3D images was performed in Meshmixer software (Autodesk) to obtain a uniform mesh density of vertices which were 1.5 mm apart from each other. Matlab (MathWorks, MA, USA) was then used to automatically annotate the left and right pupil, the pronasale, and the left and right exostomion on the aligned 
168 3D images. The landmarks were indicated on the 2D texture files automatically with a cascaded 169 convolutional network trained by Zhang et al. and transferred to the 3D images (Zang et al., 170 2016).

171 In the next step, the 3D images were cropped in order to remove excess data of the subjects' 172 head and select only the face. A general face template was scaled to every individual 3D image 173 by a Procrustes transformation based on the landmarks of the left and right pupil, the pronasale, 174 and the left and right exostomion. After the template was scaled to the individual subjects, the 175 outer boundary of the scaled template was used to crop the 3D images and to remove excess data 176 such as hair, ears, and the neck. Then, the Coherent Point Drift algorithm was used for non-rigid 177 deformation of the general face template to the mesh of the 3D images (Sang, Zhang \& Yu, 178 2013). After this non-rigid transformation of the general face template, resampling by a ray 179 casting algorithm was performed to create a uniform mesh pattern for all subjects with the same 180 number of vertices (Meulstee et al., 2017). From these uniformly resampled 3D images, average 181 faces were created for 3, 6, 9, and 12 months of age. Next, on the general face template (step 5), 182 the regions of the forehead, nose, cheeks, oral region, and chin were selected manually once 183 (Figure 1) (Hol et al., 2018). The selected regions were directly transferred to the individual 3D facial images for 3, 6, 9, and 12 months of age. Finally, all individual 3D images of UCLP patients at 3,6, 9, and 12 months of age were superimposed on the corresponding normative agematched average faces based on the children's reference frame (Brons et al., 2018). Distance maps of the 3D complete facial surface and of the nose, upper lip, chin, forehead, and cheeks were developed, and comparisons were made of distance kits for intervals of 3 to 6 months, 6 to 9 months, and 9 to 12 months of age.

\section{Statistical analysis}

Color-distance maps are presented for visual assessment of the variations in facial morphology of UCLP patients vs. control subjects. Distance maps of UCLP subjects superimposed on the corresponding normative average age-matched faces were quantified in terms of the mean distance and standard deviation for the face and its regions (nose, oral region, chin, forehead and cheeks). T-tests were applied to assess the differences in the mean distances and mean standard deviations of the distances at intervals of 3 to 6 months, 6 to 9 months, and 9 to 12 months of age 
198 for the full face and selected facial regions, with significance set at $P<0.05$. A 95\% confidence

199 interval was applied when interpreting the reliability of the results.

200

\section{Results}

202

203

204

205

206

207

208

209

210

211

212

213

214

215

216

217

218

219

220

221

222

223

224

225

Image selection

In total, 239 3D images of 30 UCLP subjects at four different time-points were available. After the selection process, 131 images were excluded because they did not meet the inclusion criteria. From the remaining 108 3D images, 34 3D images were excluded since they were duplicate images of the same patient, and 14 3D images were excluded because the corresponding image before or after primary lip closure was missing. Finally, 60 single 3D images of 20 individual patients at 3, 6, 9, and 12 months of age were included (table 1).

\section{Facial morphology of UCLP vs. control}

The average faces of UCLP subjects at 3, 6, 9, and 12 months of age are presented in Figure 2, with those at 9 and 12 months representing the post-operative state. Average faces of control subjects at similar ages were published in a previous study (Brons et al., 2018). Visual assessment of the facial morphology of UCLP vs. control subjects is presented in color-distance maps in Figure 3. At 3 months of age, the least intersurface distance between UCLP and control facial morphology was noted in the region of the eyes. The intersurface distance increased towards the unoperated cleft lip by over $-10 \mathrm{~mm}$, indicating that the average face of the UCLP subjects in this area was posterior to the average face of the control subjects. Asymmetry at the region of the nose was visible with the nostril at the affected side at $-7 \mathrm{~mm}$ compared to the average control face and that at the unaffected side $5 \mathrm{~mm}$ anterior to the location of the corresponding nostril on the average control face. At 6 months of age, the upper facial half was anterior to the average control face and nasal as well as labial asymmetry had markedly increased. At 9 months of age, the symmetry of the upper lip was completely restored despite the upper lip being an average of $4 \mathrm{~mm}$ posterior compared to the control. Nasal asymmetry, however, was not restored at this age, with the tip of the nose remaining towards the unaffected 
226 side and the left and right nostrils at $+2.5 \mathrm{~mm}$ and $-2.5 \mathrm{~mm}$, respectively, compared to the

227 average control face. At 12 months of age, the upper facial half was anterior to the average

228 control face and the symmetry of the nose improved, but asymmetry is still present with only a

229 remnant asymmetry of $2 \mathrm{~mm}$ on both sides of the nasal tip. Asymmetry of the upper lip

230 reoccurred with the unaffected side being $1 \mathrm{~mm}$ anterior to the control. At all ages, the

231 mandibular region and chin region of the UCLP subjects were posterior to the average controls

232 by 2.5 to $5 \mathrm{~mm}$.

233 Table 2 presents the mean intersurface distances between the full face and defined regions (nose,

234 upper lip, chin, forehead and cheeks) of UCLP subjects relative to the average control faces for

235 the same age. In general, the mean distance between the cleft and control was most negative at 3

236 months of age for the full face and all facial regions, ranging from $-3.8 \mathrm{~mm}$ to $-0.6 \mathrm{~mm}$, meaning

237 that the UCLP subjects on average showed retrusive facial dimensions in an anterior-posterior

238 dimension compared to the average control face of the same age. The standard deviation of the

239 mean distance for the upper lip decreased from 4.1 and $5.0 \mathrm{~mm}$ at ages 3 and 6 months,

240 respectively, to 2.5 and $2.0 \mathrm{~mm}$ at 9 and 12 months respectively, indicating a decrease in the

241 variation of the intersurface distance between UCLP patients and controls due to the primary

242 operation.

\section{Facial morphology at age intervals}

244 Table 3 shows the increments in the intersurface distances (mean, sd, p-values, and 95\% CI)

245 between UCLP subjects' faces relative to the average face of the age-matched control subjects

246 for three age intervals ( 3 to 6,6 to 9, and 9 to 12 months of age). In general, the mean increments

247 were negative from 6 to 9 months of age, indicating that during that time period, facial growth in

248 UCLP subjects was smaller than that in the averaged control faces.

249 Significant differences were found from 3 to 6 months of age for increments in the full face $(\mathrm{p}=$ $2500.01)$, nose $(p=0.02)$, and cheeks $(p=0.03)$, indicating significantly greater growth in these

251 regions in the UCLP subjects. The mean increment of the standard deviation of the upper lip

252 became significantly smaller $(\mathrm{p}=0.01)$ from 6 to 9 months of age, indicating that the shape of

253 the upper lip shows less variation between UCLP subjects and controls at 9 months of age

254 compared to 6 months. Moreover, a significant difference was found from 9 to 12 months of age

255 for the mean increment of the forehead $(\mathrm{p}=0.04)$, indicating significantly more growth of the 
256 forehead in the UCLP subjects. Finally, the mean increment of the standard deviation of the nose

257 became significantly smaller $(\mathrm{p}=0.02)$ from 9 to 12 months of age, indicating that the shape of

258 the nose shows less variation between UCLP subjects and controls.

\section{Discussion}

261 3D assessments of average facial morphology during the first year of life in children with UCLP

262 compared to non-cleft age-matched peers have not been described so far in literature. However,

263 there is one study in which the degree of 3D facial asymmetry in infants with and without

264 unilateral cleft lip and/or palate was compared to age-matched controls during the first year of

265 life (Hood et al., 2003). In the present study, facial morphology was evaluated from 3 to 12

266 months of age, including primary closure of the lip and soft palate at 6 months of age, by using

267 stereophotogrammetry. At 3 and 6 months of age, color-distance maps have shown large

268 differences in facial morphology of UCLP and control subjects in the upper lip region at the

269 location of the cleft defect and an asymmetry at the nostrils. At interval 3 to 6 months of age

270 significant increments were found in the face of UCLP subjects at the regions of the full face,

271 nose and cheeks. This may be explained by a kind of catch-up growth at age 6 months for these

272 regions, being relatively underdeveloped at age 3 months. At 9 months of age, the labial

273 symmetry was completely restored although the tip of the nose towards the unaffected side

274 showed some remnant asymmetry. At interval 6 to 9 months of age the trend seems to be that the

275 full face and facial regions grew less in the average UCLP face compared to the average control

276 face. This might be due to the impact of the surgical intervention between 6 and 9 months of age.

277 At 12 months of age, the symmetry of the nose improved, with only some remnant asymmetry

278 noted on both sides of the nasal tip. At all ages, the mandibular region and chin region of the

279 UCLP patients were 2.5-5 mm posterior to those in the average controls. Hood et al. found

280 improvement in the symmetry of the face and nose of the UCLP patients after primary surgery of

281 the lip and nose, however no improvement in symmetry of the lip was found at this interval.

282 Furthermore, they found improvement of symmetry of the lip at ages 6 to 12 months, however no

283 further improvement of symmetry of the face and nose was found. This is partially in contrast to

284 the results of the present study in which an improvement of symmetry of the lip post-surgery and

285 an improvement of nasal symmetry was found from ages 6 to 12 months. Comparing the results 
286 of both studies is challenging due to differences in the timing of the applied surgical protocols as

287 well as to differences in the methods of analyzing the 3D images.

288 After reviewing the literature on longitudinal methods for evaluation of facial growth and

289 treatment outcomes in children, we found no method for longitudinal evaluation of 3D facial

290 images of growing individuals. Interative closest point registration (ICP) is used in non-growing

291 individuals and cross sectional studies but this method is not appropriate for longitudinal

292 analysis. ICP has the effect of regression to the mean which is likely to mask any regional

293 differences in individual growth patterns. In the present study, the children's reference frame was

294 used to superimpose 3D images on the average $z$-coordinate of the right and left preauricular

295 points. In this approach, the use of the children's reference frame resembles the traditional and

296 biological registration of lateral cephalograms on the sella turcica of the cranial base.

297 Comparisons of the outcome of our study to other studies are challenging due to variations in the

298 measurement and superimposition techniques. We recommend the use of the children's reference

299 frame for cross-sectional and longitudinal evaluations of changes due to growth or treatment

300 because this is more informative than simplifying facial dimensions into inter-landmark

301 distances and angles. Registration on the children's reference frame combines changes in both

302 shape and size. Placement of the children's reference frame is a reliable technique with a

303 relatively small intra-observer error of $0.40 \mathrm{~mm}$ and an interobserver error of $0.51 \mathrm{~mm}$ (Brons et

$304 a l ., 2013$ ) and this error seems to be clinically insignificant.

305 The reproducibility of the manual identification of the facial regions was not evaluated because

306 this was performed only once on the general face template. In turn, after mapping of the general

307 face template to the individual 3D facial images, the manual selected facial regions were

308 automatically transferred from the general face template to every individual 3D facial image. We

309 used the same general face template for both the controls and the UCLP patients. Therefore,

310 intra- and inter-study reproducibility of the selection of facial regions is $100 \%$ in our case.

311 Manual selection of facial regions is likely to introduce a reproducibility error if another general

312 face template is developed with another manual selection of facial regions. However, the

313 boundaries of the facial regions have little influence on the interpretation of the color-distance 314 maps. 
315 The use of a generic mesh is a recent advancement in technical analysis of 3D facial images and

316 allows greater utilization of the potential of complex longitudinal 3D information compared to

317 simple linear and angular measurements, removes the need for localization of landmarks and

318 reduces operator error, and finally allows automatic identification of corresponding facial

319 regions.

320 Facial morphology is influenced by variations in facial expressions, even when

321 stereophotogrammetric images with a neutral facial expression are selected. The influence of

322 involuntary facial expressions has shown to range from 0.38 to $0.88 \mathrm{~mm}$ in the full facial surface,

323 but the influence is higher in the nasolabial area, especially in UCLP subjects, with mean

324 distances ranging from 0.72 to $0.93 \mathrm{~mm}$ at 3 to 12 months of age (Brons et al., 2018). This

325 variation should be taken into account when interpreting the facial morphology of individuals

326 with craniofacial malformations compared to controls of the same age or longitudinal changes of

327 facial morphology within the same group.

328 We were able to quantify deviations from the normal facial anatomy in the regions of the upper

329 lip, nose, and even the forehead at 3 and 6 months of age before lip and soft palate closure was

330 performed. The results confirm the presence of intrinsic as well as functional/adaptive

331 differences from birth on between individuals with and without UCLP as already proposed by

332 earlier researchers (Ross, 1987; Markus, Delaire \& Smith, 1992; Friede, 1998). Markus, Delaire

$333 \&$ Smith (1992) and Friede (1998) described the morpho-functional anatomy of the nasolabial

334 muscles - the anterior facial muscle chains- in patients with a complete unilateral cleft. The

335 external morphology of our 3D average cleft faces at 3 and 6 months remarkably resembles the

336 anatomy of the affected facial musculature. After primary closure of the lip and soft palate, the

337 symmetry in the upper lip is almost completely restored and the shape of the upper lip shows less

338 variation between UCLP subjects and controls, despite the upper lip being retrusive to the upper

339 lip of the average control face. This indicates that it is possible to surgically restore symmetry of

340 the upper lip, but at this early age, maxillary soft-tissue retrusion seems already developing.

341 Furthermore, the symmetry of the nose improved at 9 months of age and continued to improve

342 until 12 months of age. Longitudinal studies using stereophotogrammetry following patients and

343 controls during childhood are further needed to obtain better insights into soft-tissue changes 
344 after the first year of life as facial growth and surgical interventions may introduce improvements

345 in certain areas of the face but may also be responsible for deteriorations.

346 Stereophotogrammetry has the potential to serve as a method for early detection of facial growth

347 disturbances related to certain surgical treatments as well as soft-tissue changes related to

348 presurgical nasoalveolar molding (PNAM). It would be interesting to compare the effect of the

349 latter therapy on the nasolabial area, as the assumed effect of PNAM especially concerns the

350 shape of the nose. Stereophotogrammetry is a non-invasive and rapid imaging technique,

351 although acquisition of high-quality images is dependent on a highly experienced operator. A

352 stereophotogrammetric setup is expensive, but it provides greater value than conventional two-

353 dimensional photography by allowing quantification and localization of deviations from normal

354 facial development at an early age, as shown in this study. This enables early identification of

355 treatment protocols that are potentially detrimental for the development of normal facial

356 morphology and can be subsequently abandoned earlier. The routine use of

357 stereophotogrammetry in cleft centers is recommended in order to increase the sample size in

358 multi-center studies and for comparison of the outcomes of various cleft treatment protocols. The

359 recent development of handheld 3D cameras will stimulate the use of stereophotogrammetry in

360 the clinic due to its reduced costs and increased mobility (Kim et al., 2018).

361 With stereophotogrammetry, it is possible to detect the static morphology of the face and

362 quantify differences between multiple 3D faces in detail. However, the normal morphology can

363 only partially fulfil the desired goal of a normal facial appearance and aesthetics. Normal facial

364 dynamics is another crucial factor for perception of normal facial appearance and esthetics.

365 Therefore, future research should also focus on other standardized facial expressions besides a

366 relaxed neutral expression, like maximal smile and pouting of the lips.

367 There are limitations to our study. From our longitudinal prospective 3D facial imaging database

368 of 30 non-syndromic Caucasian UCLP subjects, 11 to 20 high-quality 3D images were included

369 per age. Initial sample size was lower than expected due to difficulty for the parents to attend at

370 every desired 3D imaging window of time. Second, despite capturing multiple 3D images per

371 occasion many 3D images had to be excluded due to not meeting the inclusion criteria. This is

372 inherent with 3D facial imaging in infants as these subjects are unable to cooperate with the ideal

373 image capture procedure. We did not differentiate between boys and girls in order to obtain a 
374 sufficient sample size. This seems to be acceptable since sexual dimorphism in the soft tissue of

375 the face in babies has not been demonstrated in literature, while on the other hand sexual

376 dimorphism in adolescents and adults has been demonstrated (Bugaighis et al., 2014). One study

377 in 3-month-old babies reported differences for some facial dimensions, but these differences

378 were related to differences in weight between subjects rather than sexual dimorphism (Kesterke

379 et al., 2016). Several studies have demonstrated the presence of craniofacial sexual dimorphism

380 in older children. One study reported sexual dimorphism in the measurements of the cranial vault

381 width and length and facial height in children at 4.7 years of age (Gaži-Čoklica et al., 1997).

382 Ferrario demonstrated differences in soft-tissue facial dimensions between boys and girls as

383 young as 6 years of age (Ferrario et al., 1999). White found a sex difference of 1-2 mm in larger

384 facial measurements such as the face height and ear-to-chin distance in infants 3 months of age,

385 but this correlated with body measurements. There were no sex differences in the nose/upper lip

386 width ratios or angular measurements, indicating there may be little sex difference in shape

387 (White et al., 2004).

388 A limitation of this study is the relatively large time window in which 3D images were acquired, 389 namely $+/-3$ weeks around the exact age of 3, 6, 9 and 12 months. The relatively large time 390 window was necessary to obtain a sufficient sample size. A difference of six weeks at most at 391 these young ages will certainly influence the study outcome. It is recommended that future 392 studies aim to limit the time window in which 3D images are made. Furthermore, future studies 393 may need to acquire 3D images every month, or even every week, to develop accurate growth 394 models for faces. The results of this study are not applicable to other cleft subtypes. Future 395 studies should focus on comparison of normative and UCLP average faces with the developing 396 facial morphology of other cleft subtypes such as unilateral cleft lip and alveolus and bilateral 397 cleft lip, alveolus, and palate.

398 Another limitation of the study is the inability to distinguish between the effect of intrinsic 399 growth and the effect of treatment in UCLP subjects. To differentiate between these two factors 400 we would need an additional control group in our study of untreated UCLP subjects. However, 401 for obvious ethical reasons it will never be possible to withhold surgical treatment from a group 402 of newborns with clefts. 


\section{Conclusions}

405 In patients with UCLP deviations from the normative average 3D facial morphology of age406 matched control subjects existed for the upper lip, nose, and even the forehead before lip and soft 407 palate closure was performed. Compared to the controls symmetry in the upper lip was restored, 408 and the shape of the upper lip showed less variation after primary lip and soft palate closure. At 409 this early age, retrusion of the soft-tissue mandible and chin, however, seems to be developing 410 already.

\section{References}

Alazzawi O, Morioka D, Miyabe M, Tosa Y, Ohkubo F, Yoshimoto S. 2017. Nasolabial growth in individuals with unilateral cleft lip and palate: A preliminary study of longitudinal observation using three-dimensional stereophotogrammetry. Journal of Craniofacial Surgery 28(5):e449e451.

Al-Rudainy D, Ju X, Mehendale F, Ayoub A. 2018. Assessment of facial asymmetry before and after the surgical repair of cleft lip in unilateral cleft lip and palate cases. International Journal of Oral and Maxillofacial Surgery 47(3):411-419.

American Cleft Palate Craniofacial Association. Standards for Approval of Cleft Palate and Craniofacial Teams Commission on Approval of Teams https://acpa-cpf.org/wpcontent/uploads/2017/06/standards.pdf; online accessed March 3, 2019. AM. 2018. Influence of involuntary facial expressions on reproducibility of 3D stereophotogrammetry in children with and without complete unilateral cleft lip and palate from 3 to 18 months of age. Clinical Oral Investigations 2019 Mar;23(3):1041-1050. doi: 10.1007/s00784-018-2520-0. Epub 2018 Jun 25. AM. Normative average faces of healthy infants during the first year of life applying uniform 3D meshes for evaluation of facial growth. PLOS One, accepted 10 May 2019. 
431 Brons S, van Beusichem ME, Bronkhorst EM, Draaisma J, Bergé SJ, Maal TJ, Kuijpers-Jagtman 432 AM. 2012. Methods to quantify soft-tissue based facial growth and treatment outcomes in 433 children: A systematic review. PLOS One 7(8):e41898.

434 Brons S, van Beusichem ME, Maal TJ, Plooij JM, Bronkhorst EM, Bergé SJ, Kuijpers-Jagtman 435 AM. 2013. Development and reproducibility of a 3D stereophotogrammetric reference frame for 436 facial soft tissue growth of babies and young children with and without orofacial clefts.

437 International Journal of Oral and Maxillofacial Surgery 42(1):2-8.

438 Bugaighis I, Tiddeman B, Mattick CR, Hobson R. 2014. 3D comparison of average faces in 439 subjects with oral clefts. European Journal of Orthodontics 36(4):365-372.

440 Chowdri NA, Darzi MA, Ashraf MM. 1990. A comparative study of surgical results with 441 rotation-advancement and triangular flap techniques in unilateral cleft lip. Journal of Plastic, 442 Reconstructive \& Aesthetic Surgery 43(5):551-556

443 Farkas LG, Hajnis K, Posnick JC. 1993. Anthropometric and anthroposcopic findings of the 444 nasal and facial region in cleft patients before and after primary lip and palate repair. The Cleft 445 Palate-Craniofacial Journal 30(1):1-12.

446 Ferrario VF, Sforza C, Poggio CE, Schmitz JH. 1999. Soft-tissue facial morphometry from 6 447 years to adulthood: a three-dimensional growth study using a new modeling. Plastic and 448 Reconstructive Surgery 103(3):768-778.

449 Friede H. 1998. Growth sites and growth mechanisms at risk in cleft lip and palate. Acta 450 Odontologica Scandinavica 56(6):346-351.

451 Gazi-Coklica V, Muretić Z, Brcić R, Kern J, Milicić A. 1997. Craniofacial parameters during 452 growth from the deciduous to permanent dentition--a longitudinal study. European Journal of 453 Orthodontics 19(6):681-689.

454 Halli R, Joshi A, Kini Y, Kharkar V, Hebbale M. 2012. Retrospective analysis of sutureless skin 455 closure in cleft lip repair. Journal of Craniofacial Surgery 23(1):e40-e44.

456 Hammond P, Hutton TJ, Allanson JE, Campbell LE, Hennekam RC, Holden S, Patton MA, 457 Shaw A, Temple IK, Trotter M, Murphy KC, Winter RM. 2004. 3D analysis of facial 458 morphology. American Journal of Medical Genetics Part A 126A(4):339-348. 
459 Hol MLF, Meulstee JW, Merks JM, Alderliesten T, Bergé SJ, Becking AG, Smeele LE, 460 Hammond P, Maal TJJ. Analyzing 3D stereophotographs: A new method for the automatic 461 segmentation of the human face into facial esthetic units using the Coherent Point Drift 462 algorithm. On behalf of the Workgroup Late Effects after Head and Neck Sarcoma, submitted for 463 publication in 2017.

464 Hood CA, Bock M, Hosey MT, Bowman A, Ayoub AF. 2003 Facial asymmetry--3D assessment 465 of infants with cleft lip \& palate. International Journal of Paediatric Dentistry 13(6):404-10.

466 Kadir A, Mossey PA, Blencowe H, Moorthie S, Lawn JE, Mastroiacovo P, Modell B. 2017.

467 Systematic review and meta-analysis of the birth prevalence of orofacial clefts in low- and 468 middle-income countries. The Cleft Palate-Craniofacial Journal 54(5):571-581.

469 Kesterke MJ, Raffensperger ZD, Heike CL, Cunningham ML, Hecht JT, Kau CH, Nidey NL, 470 Moreno LM, Wehby GL, Marazita ML, Weinberg SM. 2016. Using the 3D facial norms 471 database to investigate craniofacial sexual dimorphism in healthy children, adolescents, and 472 adults. Biology of Sex Differences 7(1):23.

473 Kim AJ, Gu D, Chandiramani R, Linjawi I, Deutsch ICK, Allareddy V, Masoud MI. 2018. 474 Accuracy and reliability of digital craniofacial measurements using a small-format, handheld 3D 475 camera. Orthodontics \& Craniofacial Research [Epub ahead of print]. doi: 10.1111/ocr.12228. 476 PubMed PMID: 29863289.

477 Kuijpers MA, Chiu YT, Nada RM, Carels CE, Fudalej PS. 2014. Three-dimensional imaging 478 methods for quantitative analysis of facial soft tissues and skeletal morphology in patients with 479 orofacial clefts: a systematic review. PLOS One 9(4):e93442.

480 Mancini L, Gibson TL, Grayson BH, Flores RL, Staffenberg D, Shetye PR. 2018. Three481 dimensional soft tissue nasal changes after nasoalveolar molding and primary cheilorhinoplasty 482 in infants with unilateral cleft lip and palate. The Cleft Palate-Craniofacial Journal 56(1):31-38. 483 Markus AF, Delaire J, Smith WP. 1992. Facial balance in cleft lip and palate. II. Cleft lip and 484 palate and secondary deformities. British Journal of Oral and Maxillofacial Surgery 30(5):296485304. 
486 Meulstee JW, Verhamme LM, Borstlap WA, Van der Heijden F, De Jong GA, Xi T, Bergé SJ, 487 Delye H, Maal TJJ. 2017. A new method for three-dimensional evaluation of the cranial shape

488 489

490

491

492

493

494

495

496

497

498

499

500

501

502

503

504

505

506

507

508

509

510

511

512

513 and the automatic identification of craniosynostosis using 3D stereophotogrammetry.

International Journal of Oral and Maxillofacial Surgery 46(7):819-826.

Morioka D, Mandrano N, Fujimoto H, Koga Y, Sato N, Tosa Y, Ohkubo F, Yoshimoto S. 2018.

Longitudinal follow-up of individuals with cleft lip using three-dimensional

stereophotogrammetry. Journal of Craniofacial Surgery 29(5):1261-1265.

Ness AR, Wills AK, Waylen A, Al-Ghatam R, Jones TE, Preston R, Ireland AJ, Persson M, Smallridge J, Hall AJ, Sell D, Sandy JR. Centralization of cleft care in the UK. Part 6: a tale of two studies. Orthod Craniofac Res. 2015 Nov;18 Suppl 2:56-62.

Reddy SG, Reddy RR, Bronkhorst EM, Prasad R, Kuijpers Jagtman AM, Bergé S. 2010.

Comparison of three incisions to repair complete unilateral cleft lip. Plastic and Reconstructive Surgery 125(4):1208-1216.

Ross RB. 1987. Treatment variables affecting facial growth in complete unilateral cleft lip and palate. The Cleft Palate Journal 24(1):5-77.

Sang Q, Zhang JZ, Yu Z. 2013. Robust non-rigid point registration based on feature-dependent finite mixture model. Pattern Recognition Letters 34(13):1557-1565.

Shaw WC, Semb G, Nelson P, Brattström V, Mølsted K, Prahl-Andersen B, Gundlach KK. 2001. The Eurocleft project 1996-2000: overview. Journal of Craniomaxillofacial Surgery 29(3):131140; discussion 141-142.

Shaw WC, Semb G, Nelson P, Brattström V, Mølsted K, Prahl-Andersen B. 2000. The Eurocleft Project 1996-2000. Amsterdam: IOS Press.

White JE, Ayoub AF, Hosey MT, Bock M, Bowman A, Bowman J, Siebert JP, Ray A. 2004. Three-dimensional facial characteristics of Caucasian infants without cleft and correlation with body measurements. The Cleft Palate-Craniofacial Journal 41(6):593-602.

World Health Organization. 2000. Global strategies to reduce the healthcare burden of craniofacial anomalies. Report of WHO Meetings on International Collaborative Research on Craniofacial Anomalies, Geneva, Switzerland. Park City, Utah, USA.

Peer) reviewing PDF | (2019:03:35537:1:1:NEW 20 May 2019) 
514 Wu J, Liang S, Shapiro L, Tse R. 2016. Measuring symmetry in children with cleft lip. Part 2:

515 quantification of nasolabial symmetry before and after cleft lip repair. The Cleft Palate-

516 Craniofacial Journal 53(6):705-713.

517 Zhang K, Zhang Z., Li Z., Qiao Y. Joint face detection and alignment using multitask cascaded 518 convolutional networks. IEEE Signal Processing Letters 23(10):1499-1503. 


\section{Figure Legends}

521 Figure 1. Selected regions for evaluation of facial growth: total facial surface, nose, upper lip, 522 chin, forehead, cheeks.

523

524 Figure 2. Average faces of UCLP subjects.

525 (A) Age 3 months.

526 (B) Age 6 months.

527 (C) Age 9 months.

528 (D) Age 12 months.

529

530 Figure 3. Visual assessment of average facial morphology of UCLP vs. controls in color531 distance maps. Color scale red - blue $=-10.0 \mathrm{~mm}-+5.0 \mathrm{~mm}$ ).

532 (A) Age 3 months.

533 (B) Age 6 months.

534 (C) Age 9 months.

535 (D) Age 12 months. 


\section{Figure 1}

Selected regions for evaluation of facial growth: total facial surface, nose, upper lip, chin, forehead, cheeks.

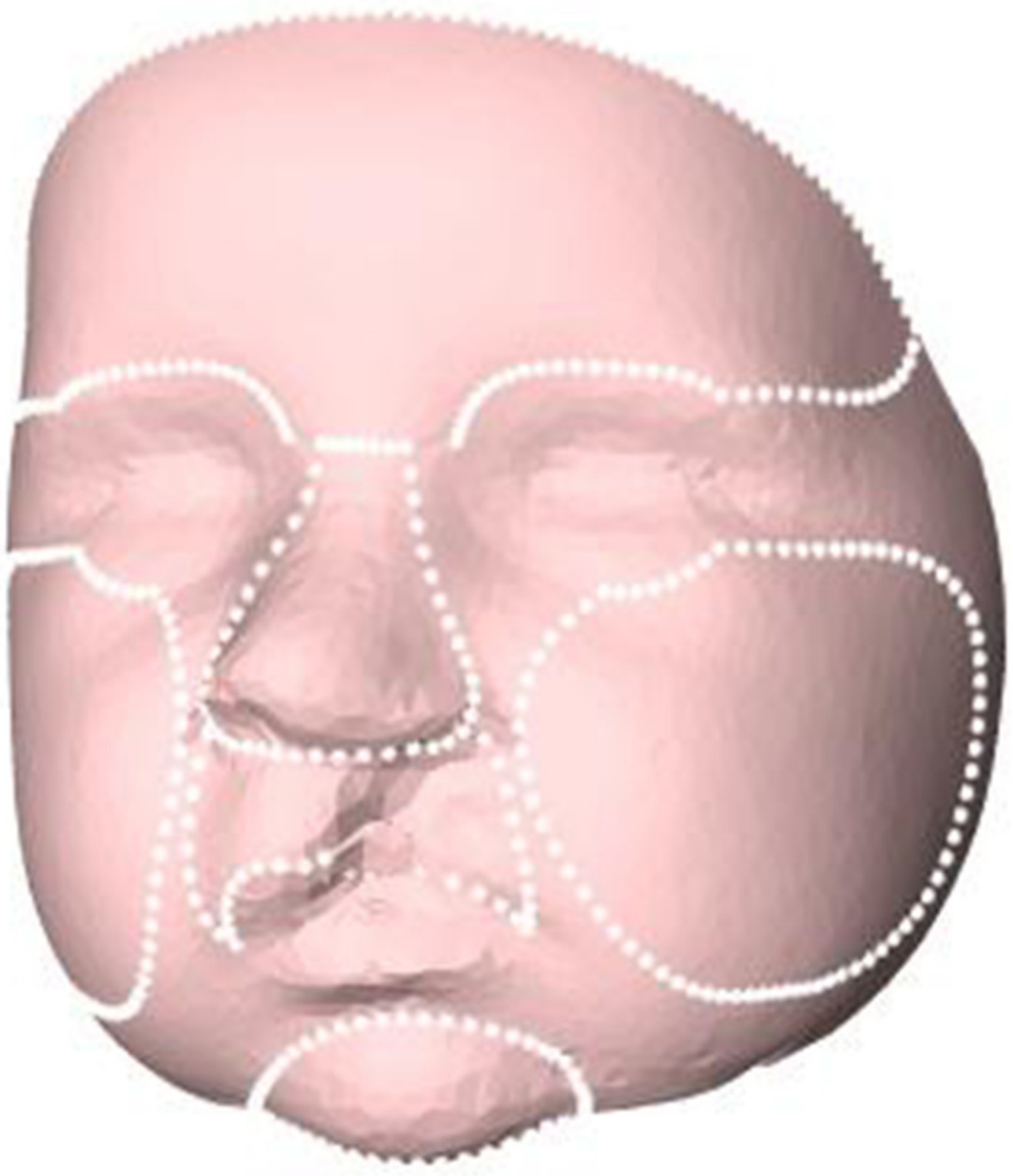


Figure 2

Average faces of UCLP subjects at age 3, 6, 9and 12 months of age. 
A

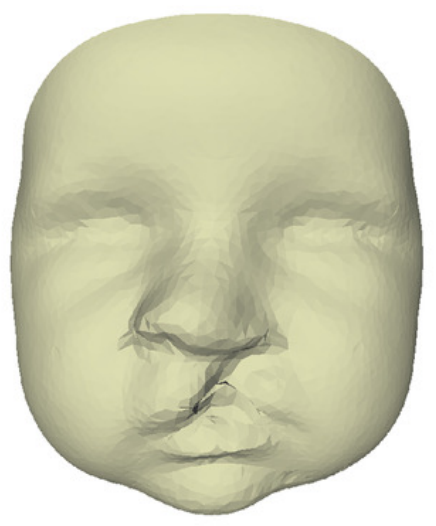

B

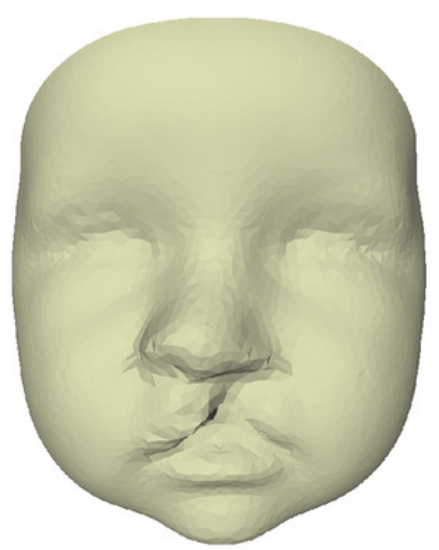

C

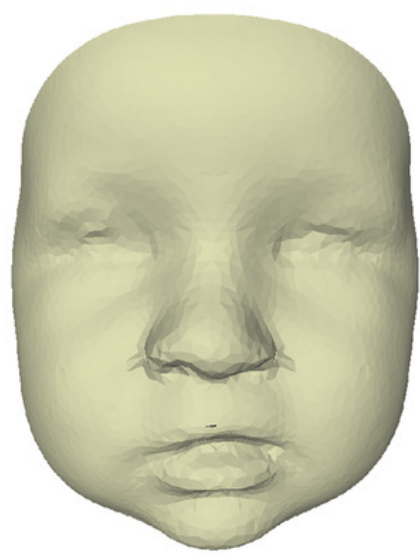

D

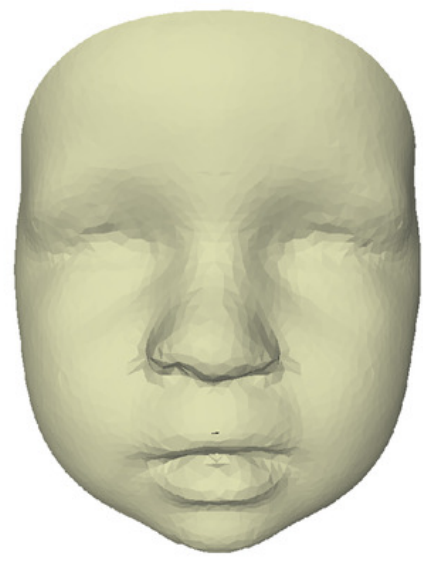

Peer) reviewing PDF | (2019:03:35537:1:1:NEW 20 May 2019) 
Figure 3

Visual assessment of average facial morphology of UCLP vs. controls atage $3,6,9$ and 12 months in colour distance map (colour scale red - blue $=-10.0 \mathrm{~mm}-+5.0 \mathrm{~mm}$ ). 
A

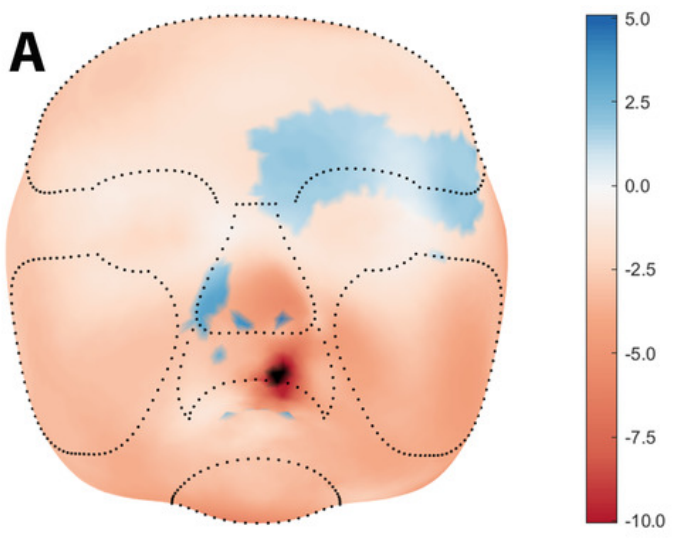

B
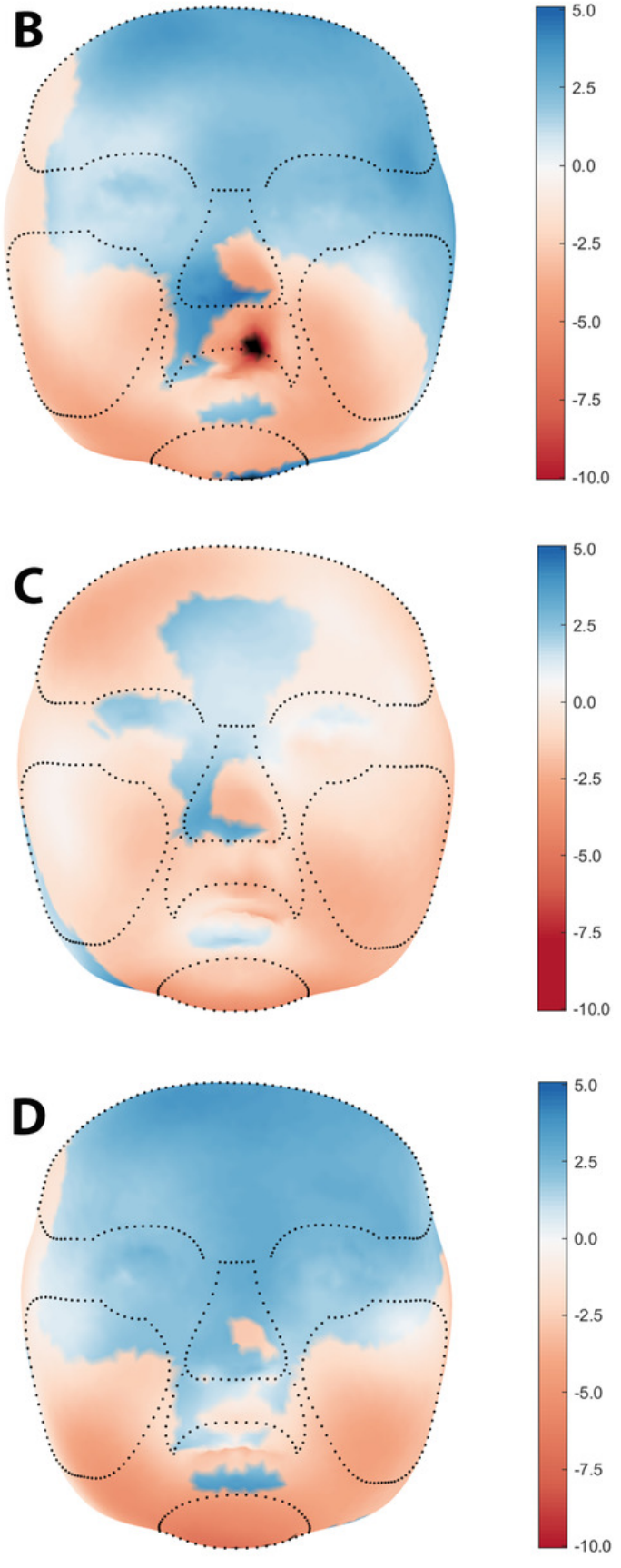


\section{Table 1 (on next page)}

Selection process forthe eligible 3D images of UCLP subjects.

UCLP, unilateral cleft lip, alveolus, and palate. 
2 Table 1. Selection process for the eligible 3D images of UCLP subjects

\begin{tabular}{l|r|r|r|r|r} 
Age & $\mathbf{3}$ mo & $\mathbf{6}$ mo & $\mathbf{9}$ mo & $\mathbf{1 2}$ mo & Total \\
\hline Children in the database $(\mathrm{n}=30)$ & 27 & 29 & 25 & 14 & \\
\hline 3D images in the database & 70 & 71 & 57 & 41 & 239 \\
\hline Excluded images (did not meet inclusion criteria) & 45 & 35 & 30 & 21 & 131 \\
\hline Exclusion of duplicate images & 6 & 11 & 10 & 7 & 34 \\
\hline Exclusion of images with missing corresponding images & 6 & 5 & 1 & 2 & 14 \\
\hline Included children ( $\mathrm{n}=20)$ & 13 & 20 & 16 & 11 & \\
\hline
\end{tabular}

3

4 UCLP, unilateral cleft lip, alveolus, and palate.

5 


\section{Table 2 (on next page)}

Mean distances andstandard deviations (in $\mathrm{mm}$ ) of the full face and regions of the nose, upperlip, chin, forehead and cheeks of UCLP subjects relative to the agematchedaverage control face.

$U C L P$, unilateral cleft lip, alveolus, and palate. 
1 Table 2. Mean distances and standard deviations (in $\mathbf{m m}$ ) of the full face and regions of the

2 nose, upper lip, chin, forehead and cheeks of UCLP subjects relative to the age-matched

3 average control face

\begin{tabular}{|c|c|c|c|c|}
\hline Region & $\mathbf{N}$ & $\begin{array}{c}\text { Age } \\
\text { (months) }\end{array}$ & $\begin{array}{l}\text { Mean } \\
(\mathrm{mm})\end{array}$ & $\begin{array}{l}\text { Std. } \\
(\mathrm{mm})\end{array}$ \\
\hline \multirow[t]{4}{*}{ Full face } & 13 & 3 & -2.5 & 5.5 \\
\hline & 20 & 6 & 0.0 & 5.2 \\
\hline & 16 & 9 & -0.9 & 4.5 \\
\hline & 11 & 12 & 0.5 & 4.2 \\
\hline \multirow[t]{4}{*}{ Nose } & 13 & 3 & -1.3 & 3.7 \\
\hline & 20 & 6 & 0.9 & 3.7 \\
\hline & 16 & 9 & -0.1 & 3.1 \\
\hline & 11 & 12 & 1.8 & 2.0 \\
\hline \multirow[t]{4}{*}{ Upper lip } & 13 & 3 & -3.7 & 4.1 \\
\hline & 20 & 6 & -2.2 & 5.0 \\
\hline & 16 & 9 & -2.2 & 2.5 \\
\hline & 11 & 12 & 0.2 & 2.0 \\
\hline \multirow[t]{4}{*}{ Chin } & 13 & 3 & -4.2 & 5.9 \\
\hline & 20 & 6 & -1.0 & 4.0 \\
\hline & 16 & 9 & -1.8 & 2.8 \\
\hline & 11 & 12 & -2.2 & 4.8 \\
\hline \multirow[t]{4}{*}{ Forehead } & 13 & 3 & -0.6 & 2.8 \\
\hline & 20 & 6 & 1.8 & 3.2 \\
\hline & 16 & 9 & -0.2 & 3.1 \\
\hline & 11 & 12 & 3.0 & 2.6 \\
\hline \multirow[t]{4}{*}{ Cheeks } & 13 & 3 & -3.8 & 4.9 \\
\hline & 20 & 6 & -1.2 & 4.1 \\
\hline & 16 & 9 & -1.6 & 3.8 \\
\hline & 11 & 12 & -1.4 & 3.3 \\
\hline
\end{tabular}

4

5

6

$7 U C L P$, unilateral cleft lip, alveolus, and palate. 


\section{Table 3 (on next page)}

T-test for equalityof increments (mean and std.) between UCLP subjects relative to the average age-matchedface of control subjects ( $p$-value) for the three age intervals.

${ }^{\text {a }}$ Significant differences in the age interval of 3-6 months in the mean difference for the full face, nose, and cheeks.

${ }^{\mathrm{b}}$ Significant differences in the age interval of 6-9 months in the mean difference for the standard deviation of the upper lip and nose.

${ }^{\text {' }}$ Significant differences in the age interval of 9-12 months in the mean difference for the forehead and the standard deviation of the nose.

$U C L P$, unilateral cleft lip, alveolus, and palate.

Std, standard deviation 
1 Table 3. T-test for equality of increments (mean and std.) between UCLP subjects relative 2 to the average age-matched face of control subjects (p-value) for the three age intervals

\begin{tabular}{l|c|c|c|c|c|c|c} 
Region & $\begin{array}{c}\text { Age } \\
\text { interval } \\
\text { (month) }\end{array}$ & $\begin{array}{c}\text { Mean } \\
\text { increment } \\
\text { mean (mm) }\end{array}$ & $\mathbf{9 5 \%}$ CI & $\begin{array}{c}\boldsymbol{p} \text {-value } \\
\text { (mean) }\end{array}$ & $\begin{array}{c}\text { Mean } \\
\text { increment } \\
\text { std. (mm) }\end{array}$ & $\mathbf{9 5 \%}$ CI & $\begin{array}{c}\boldsymbol{p} \text {-value } \\
\text { (std.) }\end{array}$ \\
\hline Full face & $3-6$ & 2.5 & $0.8-4.2$ & $0.01^{\mathrm{a}}$ & -0.3 & $-1.4 \ldots 0.8$ & 0.55 \\
\hline & $6-9$ & -1.0 & $-2.7-0.8$ & 0.27 & -0.7 & $-1.5 \ldots 0.1$ & 0.09 \\
\hline & $9-12$ & 1.4 & $-1.1-4.0$ & 0.26 & -0.2 & $-1.3 \ldots 0.8$ & 0.65 \\
\hline Nose & $3-6$ & 2.2 & $0.4-4.0$ & $0.02^{\mathrm{a}}$ & 0.0 & $-1.1 \ldots 1.1$ & 0.99 \\
\hline & $6-9$ & -1.0 & $-2.7-0.8$ & 0.27 & -0.7 & $-1,7 \ldots 0.3$ & 0.15 \\
\hline & $9-12$ & 1.9 & $-0.5-4.3$ & 0.11 & -1.0 & $-1.9 \ldots-0.2$ & $0.02^{\mathrm{c}}$ \\
\hline Upper lip & $3-6$ & 1.5 & $-0.9-4.0$ & 0.21 & 0.9 & $-0.3 \ldots 2.1$ & 0.15 \\
\hline & $6-9$ & 0.0 & $-2.3-2.3$ & 0.99 & -2.5 & $-3.5 \ldots-1.5$ & $0.01^{\mathrm{b}}$ \\
\hline & $9-12$ & 2.4 & $-0.7-5.5$ & 0.13 & -0.5 & $-1.2 \ldots 0.2$ & 0.15 \\
\hline Chin & $3-6$ & 3.2 & $-0.4-6.8$ & 0.08 & -1.9 & $-5.4 \ldots 1.6$ & 0.26 \\
\hline & $6-9$ & -0.7 & $-4.6-3.1$ & 0.70 & -1.2 & $-2.9 \ldots 0.5$ & 0.17 \\
\hline Forehead & $9-12$ & -0.4 & $-4.7-3.8$ & 0.84 & 1.9 & $-0.4 \ldots 4.3$ & 0.10 \\
\hline & $3-6$ & 2.4 & $-0.6-5.4$ & 0.11 & 0.4 & $-0.6 \ldots 1.4$ & 0.44 \\
\hline & $6-9$ & -2.0 & $-4.9-0.9$ & 0.17 & -0.1 & $-1.1 \ldots 0.9$ & 0.87 \\
\hline Cheeks & $3-12$ & 3.3 & $0.1-6.4$ & $0.04^{\mathrm{c}}$ & -0.4 & $-1.5 \ldots 0.6$ & 0.42 \\
\hline & $6-9$ & 2.6 & $0.2-5.0$ & $0.03^{\mathrm{a}}$ & -0.8 & $-2.2 \ldots 0.5$ & 0.21 \\
\hline & $9-12$ & -0.4 & $-2.8-2.0$ & 0.75 & -0.3 & $-1.3 \ldots 0.6$ & 0.50 \\
\hline & & 0.1 & $-3.1-3.4$ & 0.93 & -0.4 & $-1.5 \ldots 0.7$ & 0.42
\end{tabular}

3

4 5

a Significant differences in the age interval of 3-6 months in the mean difference for the full face, nose, and cheeks.

${ }^{b}$ Significant differences in the age interval of 6-9 months in the mean difference for the standard deviation of the upper lip and nose.

c Significant differences in the age interval of 9-12 months in the mean difference for the forehead and the standard deviation of the nose.

$U C L P$, unilateral cleft lip, alveolus, and palate.

Std, standard deviation 